\section{GW23-e1818 SEMEN CASSIAE EXTRACT REDUCES MYOCARDIAL ISCHAEMIA AND REPERFUSION INJURY IN TYPE 2 DIABETIC BUT NOT NORMAL RATS}

doi:10.1136/heartjnl-2012-302920a.95

${ }^{1}$ LV Weifeng, ${ }^{2}$ Shi Zhenwei, ${ }^{1}$ Liming Yu, ${ }^{1}$ Hongyu Oiao, ${ }^{1}$ Ping Qu, ${ }^{1}$ Ru Tie, ${ }^{1}$ Haifeng Zhang, ${ }^{1} \mathrm{Jun}$ Yu, ${ }^{1}$ Haifeng Zhang. ${ }^{1}$ Center of Teaching Experiment, Fourth Military Medical

University; ${ }^{2}$ School of Aerospace Clinical Medicine, Fourth Military Medical University

Objectives Patients with type 2 diabetes mellitus (DM), which is characterised by hyperlipidaemia, are liable to more severe and fatal myocardial infarction. Semen Cassiae is proved to reduce serum unsaturated fatty acids levels. This study was to investigate whether the Semen Cassiae extract reduces myocardial ischaemia and reperfusion $(\mathrm{MI} / \mathrm{R})$ injury, and if so, to further study the underlying mechanisms.

Methods The high-fat diet-fed streptozotocin (HFD-STZ) rat model (type 2 DM model) was developed. Age- and gendermatched normal and DM rats were given the extract mixed into the fodder of the concentration of $50 \mathrm{~g}$ crude herbal medicine $/ \mathrm{kg}$ BW for 14 days. Subsequently these animals were subjected to 30 min of myocardial ischaemia and $4 \mathrm{~h}$ of reperfusion.

Results Compared with the normal control, DM rats showed significantly increased plasma total cholesterol (TC, $4.63 \pm 0.10 \mathrm{mmol} /$ 1 vs $1.57 \pm 0.13 \mathrm{mmol} / \mathrm{l}, \mathrm{n}=8, \mathrm{p}<0.05$ ) and triglyceride (TG, 1.13 $\pm 0.15 \mathrm{mmol} / \mathrm{l}$ vs $0.68 \pm 0.09 \mathrm{mmol} / \mathrm{l}, \mathrm{p}<0.05)$. This model also had more severe $\mathrm{MI} / \mathrm{R}$ injury and cardiac functional impairment. Feeding DM rats with Semen Cassiae extract significantly reduced the plasma TC $(2.10 \pm 0.33 \mathrm{mmol} / \mathrm{l})$, TG $(0.76 \pm 0.18 \mathrm{mmol} / \mathrm{l})$, improved the instantaneous first derivation of left ventricle pressure $\left( \pm \mathrm{LV} \mathrm{dP} / \mathrm{dt}_{\max },(2854 \pm 96)\right.$ and $-(2435 \pm 98) \mathrm{mm} \mathrm{Hg} / \mathrm{s}$ vs. $(2686 \pm 87)$ and $-(2343 \pm 86) \mathrm{mm} \mathrm{Hg} / \mathrm{s}$ in DM group, $\mathrm{n}=8)$, and reduced infarct size $((42.36 \pm 9.17) \%$ vs $(56.44 \pm 10.43) \%)$, plasma creatine kinase and lactate dehydrogenase activities, and apoptotic index $((38.2 \pm 7.3) \%$ vs $(46.3 \pm 6.7) \%)$ at the end of reperfusion (all $\mathrm{p}<0.05)$. Moreover, Semen Cassiae extract treatment also increased the antiapoptotic protein Akt and ERK1/2 expression and phosphorylation levels $(n=3, p<0.05)$. Pretreatment with a PI3K inhibitor wortmannin $(1.4 \mathrm{mg} / \mathrm{kg}$, i.p., $15 \mathrm{~min}$ before ischaemia) or an ERK1/2 inhibitor PD98059 (5 mg/kg, i.p., $15 \mathrm{~min}$ before ischaemia) significantly blocked Akt and ERK1/2 phosphorylation respectively and both inhibited the cardioprotective effects induced by Semen Cassiae extract feeding. However, Semen Cassiae extract treatment did not show any effect on the plasma TC/TG levels and MI/R injury in the normal rats.
Conclusions Our data suggest that Semen Cassiae extract effectively improves myocardial function and reduces MI/R-induced injury (including apoptosis) in diabetic but not normal rats, which is possibly attributed to the reduced TC/TG levels and the triggered cell survival signalling Akt and ERK1/2. 